\title{
Differences in Clinical Presentation of Pulmonary Embolism in Older vs Younger Patients
}

\author{
Nurdan Kokturk, MD; I. Kivilcim Oguzulgen, MD; Nalan Demir, MD; \\ Koray Demirel, MD*; Numan Ekim, MD
}

\begin{abstract}
Background In order to better define the clinical characterization of pulmonary embolism (PE) in the elderly, the clinical and laboratory findings were compared in older $(\geq 65$ years old) and younger ( $<65$ years old) patients. Methods and Results The study group comprised 149 patients (58 older and 91 younger) who received a final diagnosis of PE and were retrospectively evaluated. The severity of PE was assessed by calculating the pulmonary vascular obstruction scores (PVOs) scintigraphically: PVOs $\geq 50 \%$ was defined as severe disease. Dyspnea was the most frequent symptom in both groups. Chest pain and hemoptysis were less frequent in older patients ( $48.3 \%$ vs $79.1 \%, p=0.001 ; 6.9 \%$ vs $20.9 \%, p=0.021$, respectively) whereas syncope occurred more often in the older group (27.6\% vs $9.9 \%, \mathrm{p}=0.005$ ). PVOs $\geq 50 \%$ occurred in $55.1 \%$ of older and $32.9 \%$ of younger patients (odds ratio: 1.67, 95\%confidence interval: $1.118-2.507, \mathrm{p}=0.013$ ).

Conclusions The clinical presentation of PE can be subtle or atypical in elderly patients and hence they may have more severe disease. Therefore, a high clinical suspicion is required in order to prevent delays in diagnostic work-up and initiation of appropriate treatment. (Circ J 2005; 69: 981-986)
\end{abstract}

Key Words: Elderly; Pulmonary embolism; Pulmonary vascular obstruction score (PVOs)

$\mathbf{P}$ ulmonary embolism (PE) is a major health problem associated with a significant morbidity and mortality particularly in older patients. The overall annual incidence is approximately more than 1 case per 1,000 person-years, but this increases markedly with advancing age!-4 The incidence is distributed extremely unevenly over the ages: 1 case per 1,000,000 person-years for children aged less than 15 years, 72.4 cases per 100,000 person-years for adults aged 40-54 years and 2.8 cases per 1,000 personyears for those aged $85-89$ years.,3,5

Autopsy series have shown that PE is responsible for, or at least accompanies, approximately $12 \%$ of inhospital deaths and this rate increases to $20 \%$ for the patients aged 70 years and over.,7 Kniffin et al reported a 1-year mortality for PE of $39 \%$ in patients older than 65 years, and Sakuma et al reported that the relative risk of mortality from PE was 417.76 for the patients aged over 79 years?

The high incidence of PE requires physicians to maintain a high level of suspicion in order to make a prompt diagnosis and initiate appropriate treatment, which is even more important for elderly patients who have higher mortality rates. On the other hand, physicians have to deal with the challenge of diagnosing suspected PE in elderly because older patients with PE may present with atypical clinical features in the absence of the usual indices. However, the clinical presentation of PE in the elderly population has not been extensively investigated. In the present study, the

(Received December 22, 2004; revised manuscript received May 11, 2005; accepted May 25, 2005)

Department of Pulmonary Medicine, Gazi University School of Medicine, *Department of Nuclear Medicine, Health Ministry Training and Research Hospital, Ankara, Turkey

Mailing address: Nurdan Kokturk, MD, Gazi University School of Medicine, Department of Pulmonary Medicine, Kizilirmak Sok 16/10 Kocatepe/Ankara 06640, Turkey. E-mail: nkokturk@gazi.edu.tr (nurdank@yahoo.com) hospital records of patients with documented PE were analyzed according to age, and risk factors, presenting symptoms and signs, arterial blood gas (ABG) analysis, electrocardiographic (ECG) and echocardiographic (ECHO) findings and, pulmonary vascular obstruction scores (PVOs) as a marker of severity of the disease, were compared for a better definition of the disease characteristics in the older population.

\section{Methods}

\section{Study Population and Design}

The study was carried out at Gazi University, a large teaching hospital in Ankara. Hospital records between 1998 to 2003 from the Chest Department's database were used to identify patients who had received a final diagnosis of PE, which was established according to the protocol previously published by Prospective Investigation of Pulmonary Embolism Diagnosis investigators? Patients diagnosed with pneumonia were not included in the study.

Patients who met the entry criteria were stratified into 2 groups as older ( $\geq 65$ years old) or younger $(<65$ years old $)$ patients. The risk factors for PE were defined as follows: ${ }^{10,11}$ immobilization (at least 2 days' bed rest in the 2 weeks prior to admission), pregnancy (includes postpartum period within the 3 months prior to admission), estrogen or oral contraceptive use, stroke, obesity (body mass index $>27 \mathrm{~kg} / \mathrm{m}^{2}$ ), trauma (within past 3 months), recent operation (within past 6 weeks), malignancy, recent history of long travel ( $>6 \mathrm{~h}$ within 1 week), chronic obstructive lung disease (COPD), congestive heart failure (CHF) and prior history of venous thromboembolism.

Data were obtained regarding the presence or absence of major risk factors, presenting symptoms (dyspnea, pleuritic chest pain, hemoptysis, syncope, cough and sputum), vital signs and physical examination findings (normal, crackles, 
Table 1 Clinical and Biochemical Work-up of the Patients With Pulmonary Embolism

\begin{tabular}{|c|c|c|c|}
\hline Variables & $\begin{array}{c}\text { Older patients } \\
\quad(n=58) \\
\text { mean } \pm(S D) / n\end{array}$ & $\begin{array}{c}\text { Younger patients } \\
(n=91) \\
\text { mean } \pm(S D) / n\end{array}$ & $p$ value \\
\hline Age (years) (range) & $72.7 \pm 6.1(65-86)$ & $47.8 \pm 10.3(21-64)$ & 0.001 \\
\hline$M / F(n)$ & $21 / 37$ & $45 / 46$ & 0.078 \\
\hline Body temperature $\left({ }^{\circ} \mathrm{C}\right)$ & $36.7 \pm 0.6$ & $37.1 \pm 0.9$ & 0.007 \\
\hline Pulse (beats/min) & $96 \pm 18.7$ & $98.9 \pm 17.1$ & 0.323 \\
\hline Respiratory rate (breaths/min) & $28.3 \pm 6$ & $27.3 \pm 6.5$ & 0.342 \\
\hline Systolic blood pressure $(\mathrm{mmHg})$ & $119.1 \pm 18$ & $118.5 \pm 16.1$ & 0.814 \\
\hline Diastolic blood pressure $(\mathrm{mmHg})$ & $75.3 \pm 11.6$ & $74.2 \pm 10.9$ & 0.556 \\
\hline$p H$ & $7.4 \pm 0.4$ & $7.5 \pm 0.4$ & 0.086 \\
\hline $\mathrm{PaO}_{2}(\mathrm{mmHg})$ & $59.5 \pm 9.8$ & $66.3 \pm 10.9$ & 0.001 \\
\hline $\mathrm{PCO}_{2}(\mathrm{mmHg})$ & $32.9 \pm 8.1$ & $32.1 \pm 5.1$ & 0.528 \\
\hline $\mathrm{PaO}_{2}(\%$ of predicted $)$ & $76.5 \pm 12.7$ & $77.1 \pm 12.6$ & 0.785 \\
\hline $\mathrm{SaO}_{2}(\%)$ & $90.4 \pm 5.4$ & $92.7 \pm 3.0$ & 0.004 \\
\hline$P(A-a) O_{2}$ pressure gradient & $42.7 \pm 13.4$ & $34.9 \pm 15$ & 0.002 \\
\hline Leukocyte count $/ \mathrm{mm}^{3}$ & $10,543.1 \pm 4,857.1$ & $11,542.4 \pm 6,869.8$ & 0.336 \\
\hline PVOs (\%) & $52.5 \pm 15.9$ & $44.4 \pm 14.1$ & 0.003 \\
\hline Mean PAP & $49.7 \pm 17.7$ & $41.8 \pm 16.1$ & 0.147 \\
\hline
\end{tabular}

$\mathrm{PaO}_{2}$, partial oxygen pressure; $\mathrm{PCO}_{2}$, partial carbon dioxide pressure; $\mathrm{SaO}_{2}$, oxygen saturation; $\mathrm{PVO}$, pulmonary vascular obstruction score; PAP, pulmonary arterial pressure.

rhonchi, dullness, thrombophlebitis and signs of deep venous thrombosis (DVT)). Circulatory collapse was defined as loss of consciousness or systolic blood pressure lower than $80 \mathrm{mmHg}$. The neurological status of patients with syncope was carefully checked by neurologists using brain computed tomography as required. Patients with a current neurological disorder that could affect the status of consciousness were not included in the study. Symptoms and signs of DVT were defined as leg pain, swelling, palpable cord, erythema, tenderness and Homans' sign!2 Room air $\mathrm{ABG}$ values (ion activity $(\mathrm{pH})$, partial oxygen pressure $\left(\mathrm{PaO}_{2}\right)$, partial carbon dioxide pressure $\left(\mathrm{PCO}_{2}\right)$ and, leukocyte counts were retrospectively recorded from the department's case files. Alveolar arterial oxygen pressure gradient $\left(\mathrm{P}(\mathrm{A}-\mathrm{a}) \mathrm{O}_{2}\right)$ was calculated using a standard formula ${ }^{13}$ The age-adjusted predicted values of $\mathrm{PaO}_{2}$ were calculated according to the formula of Marshall et al ${ }^{13,14}$ (mean $\mathrm{PaO}_{2}=102-0.33$ (age of years) $\mathrm{mmHg}$ ). Percentages of the predicted values of $\mathrm{PaO}_{2}$ were calculated.

ECG and ECHO were recorded in a large group of patients and those with left ventricular hypokinesia, left ventricular concentric hypertrophia and left ventricular diastolic dysfunction were excluded from the data in order to eliminate the effect of concomitant left heart failure while evaluating the relationship of PE and the ECG and ECHO findings. Any ECG abnormalities defined as compatible with PE, such as sinus tachycardia, arrhythmia, ST$\mathrm{T}$ segment changes, $\mathrm{p}$ pulmonale, right axis deviation, right bundle-branch block or $\mathrm{S}_{1} \mathrm{Q}_{3} \mathrm{~T}_{3}$, were coded as abnormal! 15,16 Any ECHO findings of PE, such as right ventricular hypokinesis or akinesis, right ventricular dilation, interventricular septal paradoxical movement and pulmonary hypertension, were coded as abnormal 17 None of the patients had patent foramen ovale.

The anatomic severity of PE was blindly and retrospectively evaluated by calculating the PVOs from the scintigraphic images, using the previously published protocol ${ }^{18}$ Briefly, each lobe was assigned a weight based on the regional distribution of pulmonary blood flow while in the supine position. For each lobe, a semi-quantitative perfusion score from 0 to $1(0,0.25,0.5,0.75$ and 1$)$ was estimated. Each lobar perfusion score was then calculated by multiplying the weight by the perfusion score. The overall perfusion score was determined by summing the 6 separate lobar perfusion scores. The percentage of vascular obstruction by perfusion scanning (PVOs) was then calculated as: PVOs $(\%)=(1-$ overall perfusion score $) \times 100$ and a PVOs $\geq 50 \%$ was defined as severe PE! ${ }^{18}$

The relation of the ECG and ECHO findings with age, the presence of syncope and PVOs was assessed in a subgroup analysis.

\section{Statistical Analysis}

SPSS for Windows (Release 10.01, Chicago, IL, USA) statistical software was used and results are given as mean \pm SD. Chi-square and Fisher's exact chi-square were performed for group comparisons. Student's t-test was used for comparison of parametric values and the Mann-Whitney U test was performed as the nonparametric test. Pearson's correlation test was used to analyze the relation between age and PVOs. A p-value less than 0.05 was considered statistically significant.

\section{Results}

A total of 149 patients (66 males, 83 females) were included in the study: 58 in the older ( $\geq 65$ years old) and 91 in the younger $(<65$ years old) group. The mean age was $72.7 \pm 6.1$ and $47.8 \pm 10.3$, respectively. Demographic data are summarized in Table 1: $83 \%$ of both groups had risk factors for PE (major risk factors are shown in Fig 1). Immobility and a past history of PE were the most common risk factors in both groups. Recurrent disease was encountered in $21(36.2 \%)$ older and $31(34.1 \%)$ of younger patients $\left(X^{2}=0.071, p=0.861\right)$, but there was a lack of detailed data on the previous treatment of these patients. At the time of the study, recurrent disease had been treated by (i) heparin infusion followed by coumarin, (ii) low-molecular weight heparin or (iii) recombinant tissue plasminogen activator in $42(82.4 \%), 8(15.3 \%)$ and, $2(3.9 \%)$ of the patients, respectively. There were $15(25.9 \%)$ and $14(15.4 \%)$ obese older and younger patients, respectively. Trauma had occurred in $3(5.2 \%)$ of the older and $12(13.2 \%)$ of the younger patients, and malignancy was present in 8 (13.8\%) 

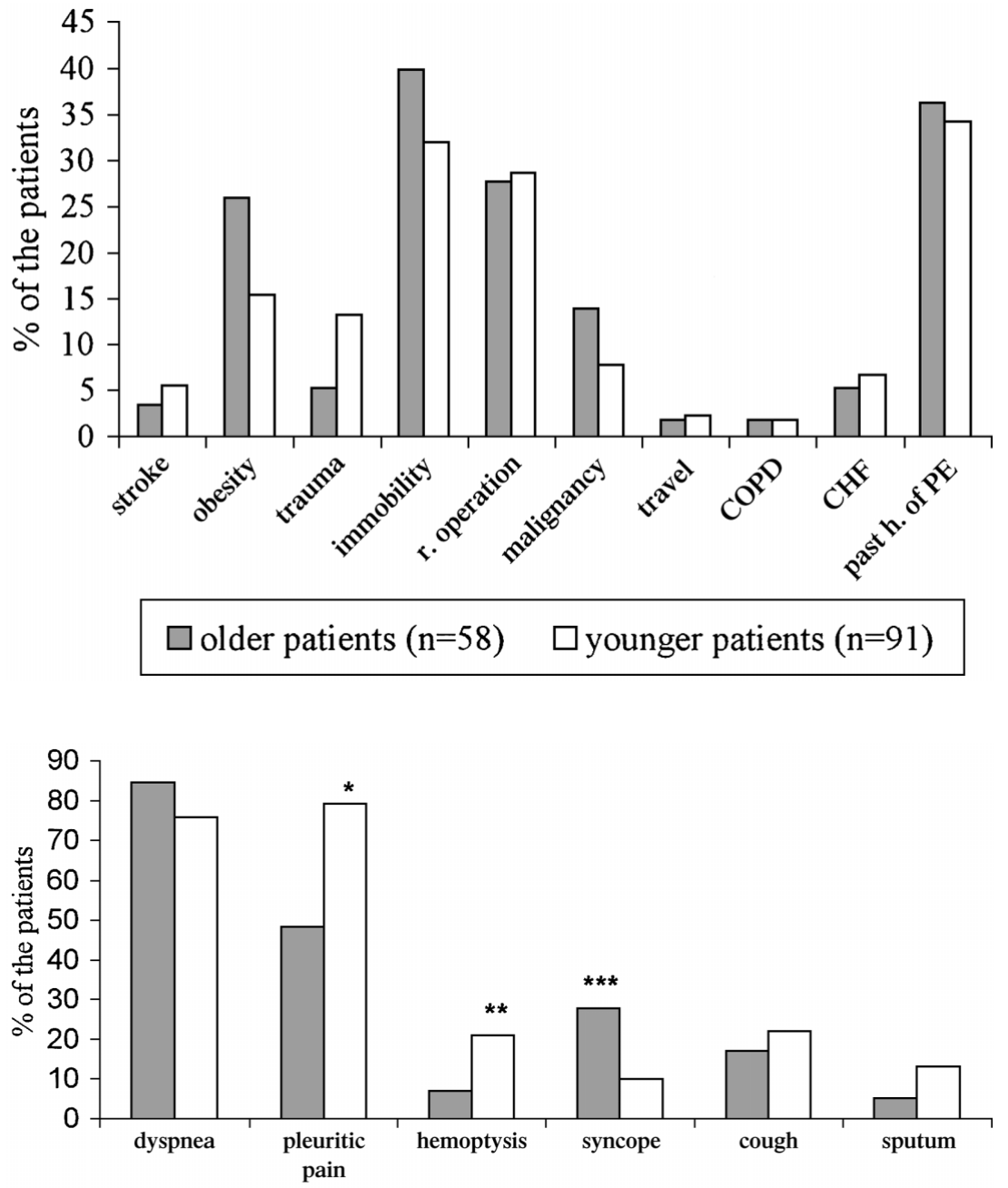

$\square$ older patients ( $n=58$ ) $\square$ younger patients $(n=91)$

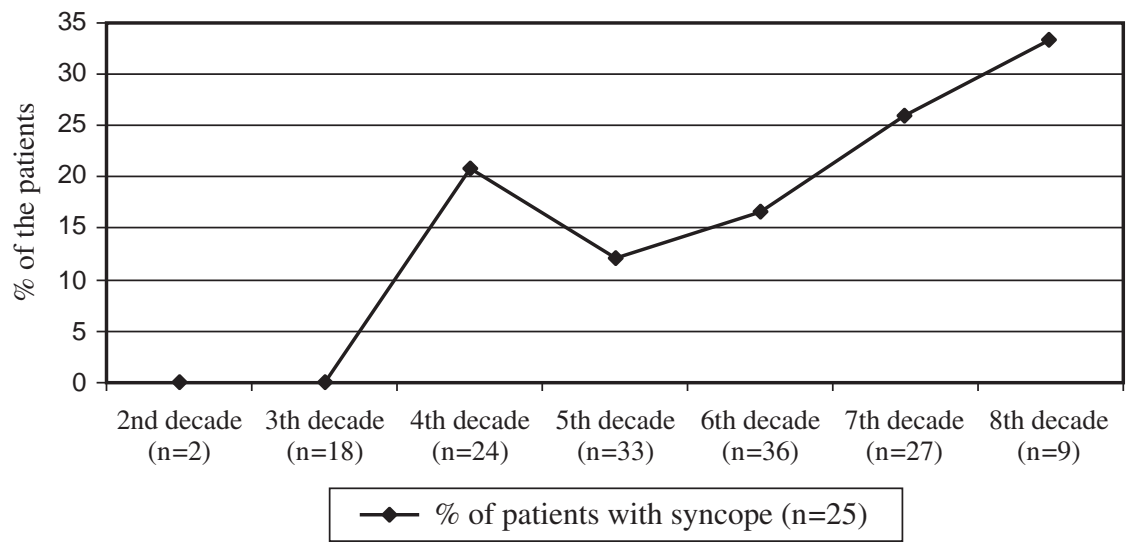

Fig 1. Risk factors for pulmonary embolism. $\mathrm{r}$, recent; h, history; PE, pulmonary embolism; $\mathrm{CHF}$, congestive heart failure. There was no statistically significant difference in distribution of risk factors.
Fig 2. Symptoms of pulmonary embolism. * $\mathrm{p}=0.001, * * \mathrm{p}=0.021, * * * \mathrm{p}=0.005$. and $7(7.7 \%)$ of the older and younger patients, respectively. The differences did not reach statistical significance. Only the younger group recorded pregnancy and the usage of oral contraceptive as risk factors in $4(4.4 \%)$ and 3 (3.3\%), respectively. There were no demonstrable risk factors in $10(17 \%)$ patients of the older group and 15 $(16 \%)$ in the younger group. All risk factors were comparable between the groups. Other comorbid conditions were also recorded: 5 patients in the older group and 2 in the younger group had diabetes mellitus and 2 patients in the younger group had nephrotic syndrome.

Presenting symptoms are illustrated in Fig 2. Dyspnea was the most common symptom in older patients whereas pleuritic chest pain was more frequent in younger patients. Dyspnea occurred in $49(84.5 \%)$ of the older and 69 $(75.8 \%)$ of the younger patients. Although the prevalence of dyspnea, cough and sputum production were equally distributed in the groups, chest pain and hemoptysis were 
Table 2 ECG and ECHO Findings of the Older and Younger Patients With Pulmonary Embolism

\begin{tabular}{|c|c|c|c|}
\hline Variables & $\begin{array}{c}\text { Older patients } \\
n(\%)\end{array}$ & $\begin{array}{c}\text { Younger patients } \\
n(\%)\end{array}$ & $p$ value $*$ \\
\hline ECG findings & $(n=44)$ & $(n=70)$ & \\
\hline At least one ECG abnormality & $22(50)$ & $24(34.3)$ & 0.096 \\
\hline Right axis deviation & $3(6.8)$ & $1(1.4)$ & 0.297 \\
\hline Atrial fibrilation & $6(13.6)$ & $7(2.9)$ & 0.053 \\
\hline Ventricular extrasystole & $2(4.5)$ & 0 & 0.147 \\
\hline ST segment changes & 0 & $1(1.4)$ & 1.000 \\
\hline Negative T waves & $6(13.6)$ & $4(5.7)$ & 0.181 \\
\hline Poor $R$ wave progression & $1(2.3)$ & $3(4.3)$ & 1.000 \\
\hline Right bundle-branch block & $2(4.5)$ & $2(2.9)$ & 0.639 \\
\hline$S_{1} Q_{3} T_{3}$ & $2(4.5)$ & $4(5.7)$ & 1.000 \\
\hline $\mathrm{ECHO}$ findings & $(n=43)$ & $(n=67)$ & \\
\hline At least one ECHO abnormality & $24(55.8)$ & $30(44.8)$ & 0.258 \\
\hline Tricuspid regurgitation & $20(46.5)$ & $26(38.8)$ & 0.452 \\
\hline$I^{\circ}$ & $14(32.5)$ & $17(25.4)$ & \\
\hline $2^{\circ}$ & $5(11.6)$ & $7(10.4)$ & \\
\hline $3^{\circ}$ & $1(2.3)$ & $2(3)$ & \\
\hline$R V$ dilatation & $16(37.2)$ & $18(26.9)$ & 0.218 \\
\hline$R V$ hypokinesia & $7(16.3)$ & $12(17.9)$ & 0.825 \\
\hline Septal paradoxical movement & $3(7)$ & 0 & 0.057 \\
\hline
\end{tabular}

*p value has been calculated by chi-square test. ECG, electrocardiographic; ECHO, echocardiographic; RV, right ventricular.

less frequent in the older group. Pleuritic chest pain occurred in $28(48.3 \%)$ and $72(79.1 \%)$ of the older and younger patients, respectively $\left(x^{2}=15.27, p=0.001\right)$. Hemoptysis was present in $4(6.9 \%)$ and $19(20.9 \%)$ of the older and younger patients, respectively $\left(\mathrm{X}^{2}=5.30, \mathrm{p}=0.021\right)$. In contrast, syncope was significantly more prevalent in the older group $(16(27.6 \%))$ than in younger $(9(9.9 \%))$ $\left(x^{2}=7.94, p=0.005\right)($ Fig 3$)$.

The evaluation of the physical examination findings revealed normal chest examination in $16(27.6 \%)$ older and $44(48.4 \%)$ younger patients. The difference was not statistically significant $(\mathrm{p}=0.068)$. Crackles were audible in $30(51.7 \%)$ of the older and $31(34.1 \%)$ of the younger patients. The presence of rhonchi and dullness were similarly distributed between the older and younger groups (1.7\%, $13.8 \%$ vs $2.2 \%, 14.3 \%$, respectively). Signs of superficial thrombophlebitis and DVT were present in $17(29.3 \%)$ and $10(17.2 \%)$ of the older, and $33(36.3 \%)$ and $15(16.4 \%)$ of the younger patients, respectively. As can be seen in Table 1, body temperature was the only vital sign that differed between the groups: older patients had a lower mean body temperature $\left(36.7 \pm 0.6^{\circ} \mathrm{C}\right.$ vs $\left.37.1 \pm 0.9^{\circ} \mathrm{C}\right)(\mathrm{p}=0.007)$.

Some representative laboratory findings are shown in Table 1. The mean $\mathrm{PaO}_{2}$ values were lower in older patients than younger patients; however, the mean percentages of the predictive values of $\mathrm{PaO}_{2}$ did not differ between the groups $(\mathrm{t}=0.274, \mathrm{p}=0.785)$. The mean $\mathrm{PCO}_{2}$ values did not differ between the groups either. All patients showed abnormal $\mathrm{P}(\mathrm{A}-\mathrm{a}) \mathrm{O}_{2}$ values, but the older patients had significantly higher mean values.

ECG findings were recorded in 131 patients: 17 patients were excluded according to the exclusion criteria; 114 and 110 patients were included for the evaluation of ECG and ECHO findings, respectively. The findings of older and younger patients were comparable (Table 2 ). The relation of syncope and the ECG and ECHO findings was also evaluated. Most of the ECG and ECHO abnormalities were higher in patients with syncope, including the frequency of at least one ECG and ECHO abnormality $(77.8 \%$ and $76.5 \%$ vs $33.3 \%$ and $44.1 \%$ in patients with and without syncope, respectively; $\mathrm{p}=0.001$ and $\mathrm{p}=0.014$ ), right axis deviation ( $16.7 \%$ vs $1 \%$ in patients with and without syncope, respectively; $\mathrm{p}=0.012)$, negative $\mathrm{T}$ waves $(22.2 \%$ vs $6.3 \%$ in patients with and without syncope, respectively; $\mathrm{p}=0.028)$, the frequency of the presence of tricuspid regurgitation $(70.6 \%$ vs $37.6 \%$ in patients with and without syncope, respectively; $\mathrm{p}=0.005)$ and right ventricular dilatation $(64.7 \%$ vs $25.8 \%$ in patients with and without syncope, respectively; $\mathrm{p}=0.002)$. PVOs were calculated in 128 patients (49 older, 79 younger) and found to be higher in older patients, indicating more severe disease. In fact, 27 of $49(55.1 \%)$ older patients and 26 of $79(32.9 \%)$ younger patients demonstrated PVOs $\geq 50 \% \quad\left(x^{2}=6.13\right.$, $\left.\mathrm{p}=0.013\right)$. Older patients had 1.67-fold risk for severe disease compared with younger patients (odds ratio: $1.67,95 \%$ confidence interval: $1.118-2.507, \mathrm{p}=0.013)$. Age showed a mild positive correlation with PVOs $(\mathrm{r}=0.24, \mathrm{p}=0.005)$.

The mean $\mathrm{PaO}_{2}$ and $\%$ predictive of $\mathrm{PaO}_{2}$ were significantly correlated with mean PVOs $(\mathrm{r}=-0.406, \mathrm{p}=0.001$, $\mathrm{r}=-0.343, \mathrm{p}=0.001$, respectively). The mean PVOs in patients with or without syncope was $86 \pm 17.7$ vs $45.6 \pm 14.1$, respectively $(\mathrm{p}=0.013)$. The frequency of the presence of at least one ECG abnormality (55\% vs $28.8 \%$, $\mathrm{p}=0.009$ ), $\mathrm{S}_{1} \mathrm{Q}_{3} \mathrm{~T}_{3}(12.5 \%$ vs $1.7 \%, \mathrm{p}=0.038)$, right ventricular dilatation $(47.5 \%$ vs $23.6 \%, \mathrm{p}=0.015)$ and right ventricular hypokinesia ( $25 \%$ vs $9.1 \%, \mathrm{p}=0.036)$ was higher in patients with higher PVOs $(\geq 50 \%)$ compared with patients with PVOs $<50 \%$.

The relation of recurrence, disease severity, and age was evaluated. Recurrent disease was encountered in 10 of $27(37 \%)$ older patients with higher PVOs compared with $27.3 \%$ in older patients with lower PVOs $X^{2}=0.52$, $\mathrm{p}=0.549$ ). Recurrent disease was encountered in 7 of 26 (26.9\%) younger patients with higher PVOs compared with $18 / 53(34 \%)$ in younger patients with lower PVOs $X^{2}=$ $0.40, \mathrm{p}=0.612$ ).

Bilateral leg vein Doppler ultrasonography (USG) was performed in 29 older and 56 younger patients: $13(44.8 \%)$ older patients and $25(44.6 \%)$ younger patients had signs of DVT on USG (no difference between the groups $X^{2}=0.000$, $\mathrm{p}=1.000$ ). Of the patients who had a pathological USG examination $78.4 \%$ also had signs of DVT on physical 
examination, whereas $21.6 \%$ of USG positive patients did not; $86.4 \%$ of the patients who displayed normal USG findings also showed normal extremities on examination $X^{2}=$ $34.331, \mathrm{p}=0.001$ ).

\section{Discussion}

It is well recognized that PE is difficult to diagnose because of its subtle and nonspecific presentation, particularly in the elderly population. Taubman et al found in a postmortem study conducted over a 6-year period that the incidence of PE was $12.8 \%$ in the elderly population and only one third were diagnosed antemortem. This underlines the need for more clinical characterization of the presentation of the disease in elderly. The higher incidence of the disease in elderly has been attributed to the accumulation of common risk factors with age. The common risk factors such as malignancy, surgery and immobilization are more prevalent in the elderly, ${ }^{19,20}$ whereas in younger patients, genetic factors, pregnancy, puerperium, estrogen and oral contraceptives, and a history of trauma are more frequent $5,11,20,21$ Some age-related conditions, for instance decreased mobility associated with decreased muscle tone and degenerative vascular changes, may also contribute to the disease development in elderly patients 22

In our study, even though it did not reach statistical significance, in agreement with previous studies immobilization and malignancy were slightly more prevalent in the elderly, whereas a history of trauma was more frequent in the younger patients. Recurrence rate would be different by age and could be related to disease severity and disease onset ${ }^{23}$ Accordingly, we analyzed the recurrence rate in both groups and in those with higher and lower PVOs. There was no statistical difference in these subgroup analyses.

Symptomatology may have a particular importance before ordering diagnostic tests. Most studies report that dyspnea and pleuritic chest pain are the most common symptoms, ${ }^{19,20,24}$ but although dyspnea was the most prevalent symptom in both groups in our study; interestingly enough, pleuritic chest pain and hemoptysis were seen less and syncope seen more in the older patients when compared with the younger ones. Pleuritic chest pain was found to be less prevalent in 2 previous studies, 20,24 in contrast to others 19,25 Two studies in which the age cut-off point was 40 years, the presenting symptoms were not found to be different in younger patients compared with older controls!1,21 The lower occurrence of chest pain in the elderly was attributed to diminished visceral pain sensation and reduced perception. ${ }^{4}$

Hemoptysis was found much less frequently in the elderly than in the younger patients, which was a similar finding to that of Stein et $\mathrm{al}^{11}$ and Timmons et $\mathrm{al}^{19}{ }^{19}$ but in contrast to Green et $\mathrm{al}^{20}{ }^{2}$ Ramos et $\mathrm{al}^{24}$ and Gisselbrecht et $\mathrm{al}^{25}$ who all showed a comparable prevalence of hemoptysis. This finding would be interpreted as a sign of infarction, which could reflect less severe disease 26

Syncope was 3-fold more common in the older patients. Stein et al demonstrated syncope as a presenting syndrome in only $8 \%$ of a total of 150 patients with documented PE, but it is interesting to see that the mean age in their subgroup was 66 years old and they showed more severe disease ${ }^{26}$ Although we did not evaluate the predominance of presenting symptoms or identify isolated cases of syncope, we can conclude that the symptom of syncope should attract more attention in elderly patients even though it is uncommon in the general PE population. The greater incidence of syncope in older patients basically could reflect reduced cardiopulmonary reserve and severe disease ${ }^{24}$ associated with hypotension, severe tachycardia, and ECG and ECHO abnormalities. Although the mean blood pressure, heart rate, and the presence of ECG and ECHO abnormalities were not different between the age groups, they were different when the patients were grouped with or without syncope.

Other indices related to severity, such as mean $\mathrm{PaO}_{2}$ and $\mathrm{SaO}_{2}$ and $\mathrm{P}(\mathrm{A}-\mathrm{a}) \mathrm{O}_{2}$ gradient, were lower in the older group, which is in agreement with most of the previous studies! 11,19,20,24 Gisselbrecht et al, however, did not demonstrate a difference in patients $>75$ years old vs $<75$ years old with massive PE $2^{25}$ Most of the previous studies did not adjust the measured $\mathrm{PaO}_{2}$ values according to age, so we calculated the predicted values of $\mathrm{PaO}_{2}$ and measured the $\%$ of predicted values. These calculated values did not differ between the groups, which underlies the importance of considering the predictive values in the interpretation of related findings. Although some comorbid conditions, such as COPD and CHF, could affect $\mathrm{PaO}_{2}$, these conditions were found to be equally distributed in the groups in our study. Hence, patients with pneumonia were excluded from the study during chart review.

Anatomic severity calculated by perfusion lung scans (ie, PVOs) has been shown to be well correlated with angiographic severity (Miller index) and the overall agreement between the 2 techniques is excellent $(r=0.82$, mean absolute difference $=2.8 \%) !^{18}$ To our knowledge, the relationship between PVOs and age has been studied in only one previous study in which they compared initial PVOs in 2 groups ( $>75$ years old vs $<75$ years old) in a subgroup of massivePE and found no difference ${ }^{25}$ Our study indicated that the mean PVOs positively correlated with age and severe PVOs ( $\geq 50 \%)$ were more prevalent in older patients. Higher PVOs was associated with deterioration in ABG values and the presence of syncope. Hence, ECG and ECHO abnormalities that could indicate more severe disease, such as $\mathrm{S}_{1} \mathrm{Q}_{3} \mathrm{~T}_{3}$ and right ventricular hypokinesia, and these were more common in patients with greater PVOs.

Our findings support the idea that PE can more severe despite the subtle symptomatology in older patients. Therefore, it is very important to identify elderly patients presenting with an atypical clinical condition who should undergo a diagnostic work-up and who are potentially treatable with the appropriate regimen.

\section{Study Limitations}

The data presented here were retrospectively collected and could have selection bias depending on the referral pattern to our institution. Hence, more severe patients would be more likely to be referred for a perfusion lung scan. Finally, the presented data lack outcome information, which would be very informative about the burden of disease in a specific population. Large prospective trials that cover the findings of chest X-ray, electrocardiography and echocardiograpy and the patients' outcomes are needed in order to draw definite conclusions.

In conclusion, this study demonstrated that the clinical presentation of PE could be subtle or atypical in elderly patients. Chest pain and hemoptysis may be absent and syncope may be more prominent in older patients compared with younger ones. Hence, such patients could have 
more severe disease and a high clinical suspicion is required in order to prevent delays in diagnostic work-up and initiation of appropriate treatment.

\section{References}

1. Silverstein MD, Heit JA, Mohr DN, Petterson TM, O'Fallon WM, Melton III LJ. Trends in the incidence of deep vein thrombosis and pulmonary embolism: A 25-year population-based study. Arch Intern Med 1998; 158: 585-593.

2. Heit JA. Risk factors for venous thromboembolism. Clin Chest Med 2003; 24: 1-12.

3. Kniffin WD, Baron JA, Barrett J, Birkmeyer JD, Anderson FA. The epidemiology of diagnosed pulmonary embolism and deep venous thrombosis in the elderly. Arch Intern Med 1994; 154: 861-866.

4. Alikhan R, Cohen AT, Combe S, Samama MM, Desjardins L, Eldor A, et al. Risk factors for venous thromboembolism in hospitalized patients with acute medical illness: Analysis of the MEDENOX study. Arch Intern Med 2004; 164: 963-968.

5. Rosendaal FR. Thrombosis in the young: Epidemiology and risk factors: A focus on venous thrombosis. Thromb Haemost 1997; 78: $1-6$.

6. Taubman LB, Silverstone FA. Autopsy proven pulmonary embolism among institutionalized elderly. J Am Geriatr Soc 1986; 34: 752 756.

7. Coon WW. The spectrum of pulmonary embolism, twenty years later. Arch Surg 1976; 111: 398-402.

8. Sakuma M, Konno Y, Shirato K. Increasing mortality from pulmonary embolism in Japan, 1951-2000. Circ J 2002; 66: 1144-1149.

9. PIOPED Investigators. Value of the ventilation/perfusion scan in acute pulmonary embolism: Results of the Prospective Investigation of Pulmonary Embolism Diagnosis (PIOPED). JAMA 1990; 263: $2753-2759$.

10. Bell WR, Simon TL. Current status of pulmonary thromboembolic disease: Pathophysiology, diagnosis, prevention and treatment. $\mathrm{Am}$ Heart J 1982; 103: 239-262.

11. Green RM, Meyer TJ, Dunn M, Glassroth J. Pulmonary embolism in younger adults. Chest 1992; 101: 1507-1511.

12. Stein PD, Terrin M, Hales CA, Palevsky HI, Saltzman HA, Thompson BT, et al. Clinical, laboratory, roentgenographic, and electrocardiographic findings in patients with acute pulmonary embolism and no pre-existing cardiac or pulmonary disease. Chest 1991; 100: 598
603.

13. Nunn JF. Applied respiratory physiology, 2nd edn. London: Whitefriars Press Ltd; 1977.

14. Marshall BE, Wyche MQ. Hypoxemia during and after anesthesia. Anesthesiology 1972; 37: 178-209.

15. Stein PD, Dalen JI, Mclntyre KM, Sasahara AA, Wenger NK, Willis $\mathrm{PW}$. The electrocardiogram in acute pulmonary embolism. Prog Cardiovasc Dis 1975; 17: 247-257.

16. Yoshinaga T, Ikeda S, Shikuwa M, Miyahara Y, Kohno S. Relationship between ECG findings and pulmonary artery pressure in patients with acute massive pulmonary thromboembolism. Circ $J$ 2003; 67: 229-232.

17. Goldhaber SZ. Pulmonary embolism. N Engl J Med 1998; 339: $93-$ 104.

18. Meyer G, Collignon MA, Guinet F, Jeffrey AA, Barritault L, Sors H. Comparison of perfusion lung scanning and angiography in the estimation of vascular obstruction in acute pulmonary embolism. Eur $J$ Nuc Med 1990; 17: 315-319.

19. Stein PD, Gottschalk A, Saltzman HA, Terrin ML. Diagnosis Of acute pulmonary embolism in the elderly. J Am Coll Cardiol 1991; 18: $1452-1457$.

20. Ramos A, Murillas J, Mascias C, Carretero MB, Portro JL. Influence of age on clinical presentation of acute pulmonary embolism. Arch Gerontol Geriatr 2000; 30: 189-198.

21. Arima M, Kanoh T, Takagi A, Tanimoto K, Oigawa T, Matsuda S. Clinical features of acute pulmonary thromboembolism in younger patients. Circ J 2003; 67: 330-333.

22. Rosendaal FR. Venous thrombosis: A multicausal disease. Lancet 1999; 353: 1167-1173.

23. Yamamoto T, Sato N, Tajima H, Takagi H, Morita N, Akutsu K, et al. Differences in the clinical course of acute massive and submassive pulmonary embolism: In hospital onset vs out of hospital onset. Circ J 2004; 68: $988-992$.

24. Timmons S, Kingston M, Hussain M, Kelly H, Liston R. Pulmonary embolism: Differences in presentation between older and younger patients. Age Ageing 2003; 32: 601-605.

25. Gisselbrecht M, Dielh JL, Meyer G, Collignon MA. Clinical presentation and results of thrombolytic therapy in older patients with massive pulmonary embolism: A comparison with non-elderly patients. J Am Geriatr Soc 1996; 44: 189-193.

26. Stein PD, Henry JW. Clinical characteristics of patients with acute pulmonary embolism stratified according to their presenting syndromes. Chest 1997; 112: 974-979. 